\title{
Capacitor physics in ultra-near-field heat transfer
}

\author{
Jian-Sheng Wang and Jiebin Peng \\ Department of Physics, National University of Singapore, Singapore 117551, Republic of Singapore
}

PACS $05.60 . \mathrm{Gg}$ - quantum transport

PACS 44.40.+a - thermal radiation

\begin{abstract}
Using the nonequilibrium Green's function (NEGF) formalism, we propose a microscopic theory for near-field heat transfer between charged metal plates focusing on the Coulomb interactions. Tight-binding models for the electrons are coupled to the electromagnetic field continuum through a scalar potential. Our approach differs from the established ones based on Rytov fluctuational electrodynamics, which deals with the transverse radiative field and vector potential. For a two quantum-dot model a new length scale emerges below which the heat current exhibits great enhancement. This length scale is related to the physics of parallel plate capacitors. At long distances $d$, the energy flux decreases as $1 / d^{2}$.
\end{abstract}

The thermal radiation in a cavity can be well-described by Planck's theory of black-body radiation [1] - a great achievement of twentieth century physics, which started , the quantum physics revolution. Two plates at temperatures $T_{0}$ and $T_{1}$ will transfer radiative heat at a rate proportional to $T_{0}^{4}-T_{1}^{4}$ in the black-body limit, follow, ing the Stefan-Boltzmann law. In the 70s both theoretical , 2] and experimental [3] 5] work have indicated corrections to the far field prediction when the distances between the plates are comparable to the thermal wavelengths of the ' electromagnetic fields. Near-field effects can be as large as a thousand fold that of the far field results [6] 8 .

Most recently, due to great progress in technology and ' precision measurements, much closer proximity is possible, in the scale of nanometers, or near contact. The near' field enhancement reported experimentally in Refs. 9, 10 ' is consistent with the established theory. However, the result in [11 is much too large to be explained by existing theories. Are there other mechanisms for the near-field effect?

Polder and van Hove $(\mathrm{PvH})$ 2] were the first to give a quantitative theory of near-field radiation using the Rytov formulation of fluctuating electromagnetic fields [12, 13. The current-current correlation is assumed to follow the equilibrium fluctuation-dissipation theorem. The average value of the Poynting vector is computed from the solution of macroscopic Maxwell equations. In this picture, the near-field contribution is largely due to evanescent modes which are absent in the far field. A quantum electrodynamics treatment with linear media and NEGF reproduces the $\mathrm{PvH}$ theory [14].

The large near-field effect is recently explained by phonon tunneling or surface phonon polaritons 15 17. In these work, although the Coulomb interaction is dealt with indirectly, it is still treated as dipole-dipole fluctuations in a charge-neutral system. We advocate that charge-charge fluctuations are important features at short distances. The aim of this letter is to propose such a fundamental theory. We begin with a tight-binding model of the electrons, as in a metal, for example, and couple it to the scalar field in a quantized form. While the electrons are on discrete lattices, the electromagnetic field is continuous and permeates the whole space. Due to the scalar field nature, we need to use Lorentz gauge quantization [18, and then take the limit of the speed of light $c$ going to infinity in order to be consistent with the gauge condition.

To demonstrate the basic idea, we define a toy model for a nano-sized capacitor consisting of two quantum dots and a one-dimensional (1D) scalar field, mediating the Coulomb interactions of the charges. The same formulation can be applied to more realistic models, such as two graphene sheets. Figure 1 illustrates the model.

We imagine a nanoscale parallel plate capacitor for which each of the plates can have a charge of 0 or $-Q$. The plates located at $z=0$ and $d$ are connected to their respective electron baths so that their charges can fluctuate. The baths are necessary ingredient so that infinite amount of energy can be transferred from one dot to the other given time. The couplings of the quantum dots with the baths are bilinear in the system and bath fermion op- 


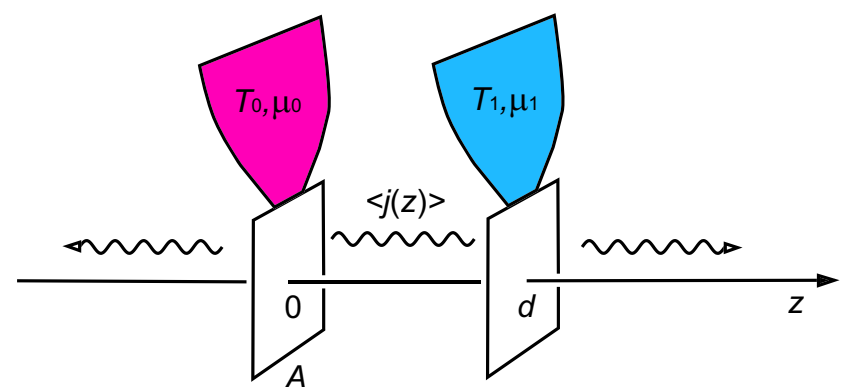

Fig. 1: Schematic diagram of the 1D quantum-dot model.

erators; their effects are captured by the self-energies of the baths 19 . We ignore the vector potential and consider the scalar potential $\phi(z)$ defined for all $z$. For short distances, the scalar potential, giving rise to the Coulomb interaction [20, is more important. The quantum of the field is known as the scalar photon 21. The Hamiltonian of the whole setup, $H=H_{\gamma}+H_{e}+H_{\text {int }}$, is

$$
\begin{aligned}
H_{\gamma} & =-s \int_{-L / 2}^{L / 2} d z \frac{1}{2}\left[\dot{\phi}^{2}+c^{2}\left(\frac{\partial \phi}{\partial z}\right)^{2}\right], \\
H_{e} & =v_{0} c_{0}^{\dagger} c_{0}+v_{1} c_{1}^{\dagger} c_{1}+\text { baths \& couplings, } \\
H_{\mathrm{int}} & =(-Q) c_{0}^{\dagger} c_{0} \phi(0)+(-Q) c_{1}^{\dagger} c_{1} \phi(d),
\end{aligned}
$$

where $s=A \epsilon_{0} / c^{2}$ is a scale factor to give $H_{\gamma}$ the dimensions of energy; $A$ is the area of the capacitor, $\epsilon_{0}$ is the vacuum permittivity, and $c$ is the speed of light. $c_{0}, c_{1}$, and their hermitian conjugates are fermionic annihilation and creation operators. The photon field can be expressed as (in the interaction picture)

$$
\phi(z, t)=\sum_{q} \sqrt{\frac{\hbar}{2 \omega_{q} s L}}\left(a_{q} e^{i\left(q z-\omega_{q} t\right)}+\text { h.c. }\right),
$$

where $\omega_{q}=c|q|$ is the photon dispersion relation, with the wavenumber $q=2 \pi k / L, k$ an integer, $a_{q}$ the bosonic annihilation operator of a photon of mode $q$, and h.c. stands for the hermitian conjugate of the preceding term. The scalar photon satisfies the unusual commutation relation, $\left[a_{q}, a_{p}^{\dagger}\right]=-\delta_{q p}$. We will take the limits $L \rightarrow \infty$ and $c \rightarrow \infty$ at the end of calculation. The latter reproduces Poisson's equation for the field. We should regard this limiting procedure as only a calculational technique; an alternative and equivalent method based on Joule heating is also possible [20].

Our task is to compute the energy current between the dots. From continuity requirements of the field energy, we can establish an expression for the current density operator to be $\epsilon_{0} \dot{\phi} \partial \phi / \partial z$. However, to obtain a correct quantum version of the operator, we need to symmetrize the two factors and also demand anti-normal order [22,23] (denoted by the bars and colons here):

$$
j=\frac{\epsilon_{0}}{2}\left[\left|: \dot{\phi} \frac{\partial \phi}{\partial z}:\right|+\left|: \frac{\partial \phi}{\partial z} \dot{\phi}:\right|\right] .
$$

Anti-normal order dictates that we swap the annihilation operator to the left of the creation operator if that is not already the case. This removes the zero-point motion contribution. We can relate the expectation value of $j$ to the Green's functions of the photons. The end effect of the operator ordering is to take only the positive frequency contribution of the Green's function (a justification depends on omitting correlations between annihilation-annihilation operators, and similarly creation-creation operators, and will be presented elsewhere [24]). The average energy current per unit area at location $z$ can be obtained from

$$
\langle j(z)\rangle=\left.\epsilon_{0} \int_{0}^{\infty} \frac{d \omega}{\pi} \hbar \omega \operatorname{Re} \frac{\partial D^{>}\left(\omega, z, z^{\prime}\right)}{\partial z^{\prime}}\right|_{z^{\prime}=z},
$$

where $D^{>}\left(\omega, z, z^{\prime}\right)=\int_{-\infty}^{+\infty} D^{>}\left(z, t ; z^{\prime}, 0\right) e^{i \omega t} d t$ is the frequency domain greater Green's function for the field $\phi$.

We evoke the machinery of NEGF [25 28] to calculate the required Green's functions. First, we define the contour-ordered Green's function as

$$
D\left(z, \tau ; z^{\prime}, \tau^{\prime}\right)=-\frac{i}{\hbar}\left\langle T_{\tau} \Delta \phi(z, \tau) \Delta \phi\left(z^{\prime}, \tau^{\prime}\right)\right\rangle_{\text {noneq }}
$$

where $\tau$ and $\tau^{\prime}$ are Keldysh contour times, $T_{\tau}$ is the contour order operator, and the average is over a nonequilibrium steady state, $\Delta \phi(z, \tau)=\phi(z, \tau)-\langle\phi(z, \tau)\rangle_{\text {noneq }}$. The operators are in the Heisenberg picture. Transforming into the interaction picture, and using the standard diagrammatic expansion [29, we can summarize the result in a contour-ordered Dyson equation, which can be organized as a pair of equations in real time, the retarded Dyson equation and the Keldysh equation. Due to time translational invariance, the equations become simple in the frequency domain, given as, for the Keldysh equation,

$$
D^{>}\left(\omega, z, z^{\prime}\right)=\sum_{j, k=0,1} D^{r}\left(\omega, z, z_{j}\right) \Pi_{j k}^{>}(\omega) D^{a}\left(\omega, z_{k}, z^{\prime}\right),
$$

where $z_{0}=0, z_{1}=d$. The retarded Green's function satisfies

$$
\begin{aligned}
D^{r}\left(\omega, z, z^{\prime}\right)= & D_{0}^{r}\left(\omega, z, z^{\prime}\right)+ \\
& \sum_{j, k=0,1} D_{0}^{r}\left(\omega, z, z_{j}\right) \Pi_{j k}^{r}(\omega) D^{r}\left(\omega, z_{k}, z^{\prime}\right),(9)
\end{aligned}
$$

where, $D_{0}^{r}\left(\omega, z, z^{\prime}\right)=-e^{i \frac{\omega}{c}\left|z-z^{\prime}\right|} /\left(2 i \epsilon_{0} A \omega / c\right)$, is the free photon retarded Green's function. The advanced Green's function is obtained by symmetry, $D^{a}\left(\omega, z, z^{\prime}\right)=$ $D^{r}\left(\omega, z^{\prime}, z\right)^{*}$. We also have the identity $D^{>}-D^{<}=$ $D^{r}-D^{a}$. To make contact with the usual dyadic Green's function method [8], one can turn the Dyson equation into a differential equation by operating with the inverse of the free Green's function. However, due to the discrete nature of the problem, $z_{j}$ takes only a finite set of values. The above equation (9) can be solved directly, by choosing a finite set of values $\{0, d, z, \cdots\}$. It becomes a system of linear equations. 
In addition to the Green's functions of the photons, we also need the Green's functions of the electrons to compute the photon self energies. A similar Dyson equation for the electrons can be established, with the Green's function [25] $G_{j k}\left(\tau, \tau^{\prime}\right)=-\frac{i}{\hbar}\left\langle T_{\tau} c_{j}(\tau) c_{k}^{\dagger}\left(\tau^{\prime}\right)\right\rangle$ and electron selfenergy $\Sigma$. The problem is completely specified if these self-energies are known. However, for interacting systems like the electron-photon interaction $H_{\mathrm{int}}$, no simple closed form is possible (except the formal Hedin equations [30]). For the two-dot model, we present a calculation with the self-consistent Born approximation (SCBA) 29]. In this framework the photon self-energy due to the electronphoton interactions is essentially charge-charge correlation, in contour time, as $(j, k=0,1)$

$$
\begin{aligned}
\Pi_{j k}\left(\tau, \tau^{\prime}\right) & =-\frac{i}{\hbar}\left\langle T_{\tau} q_{j}(\tau) q_{k}\left(\tau^{\prime}\right)\right\rangle \\
& \approx-i \hbar Q^{2} G_{j k}\left(\tau, \tau^{\prime}\right) G_{k j}\left(\tau^{\prime}, \tau\right)
\end{aligned}
$$

where $q_{j}=(-Q) c_{j}^{\dagger} c_{j}$, and the second line is obtained by applying Wick's theorem. The appearance of the selfenergy $\Pi$ which is also the charge susceptibility (linear response of the induced charge by applied potential) underlines the difference between the present theory and the standard $\mathrm{PvH}$, which relies on the current-current correlation, or frequency-dependent dielectric function. The contour expression can be used to derive the real-time formula 25, e.g., the retarded one in the frequency domain needed for solving the Dyson equation is

$$
\begin{aligned}
\Pi_{j k}^{r}(\omega)= & -i \hbar Q^{2} \int_{-\infty}^{+\infty} \frac{d E}{2 \pi \hbar}\left[G_{j k}^{r}(E) G_{k j}^{<}(E-\hbar \omega)\right. \\
& \left.+G_{j k}^{<}(E) G_{k j}^{a}(E-\hbar \omega)\right] .
\end{aligned}
$$

These formulas above are general. Since the electrons cannot jump from the left lead to the right lead, the offdiagonal elements of the self energies are zero; we only have nonzero diagonal terms $\Pi_{j j}$. The electron retarded Green's function is given by $G_{j j}^{r}(E)=1 /\left(E-v_{j}-\Sigma_{j}^{r}(E)-\right.$ $\left.\Sigma_{\mathrm{n}, j}^{r}(E)\right)$, where the bath contribution to the self-energy is chosen to follow the Lorentz-Drude model [19], $\Sigma_{j}^{r}(E)=$ $\frac{1}{2} \Gamma_{j} /\left(i+E / E_{j}\right)$, where $\Gamma_{j}$ and $E_{j}$ are the bath model constants. The lesser Green's function is given by a Keldysh equation, $G_{j j}^{<}(E)=G_{j j}^{r}(E)\left(\Sigma_{j}^{<}(E)+\Sigma_{\mathrm{n}, j}^{<}(E)\right) G_{j j}^{a}(E)$. The lesser components of the bath self energies follow from the fluctuation-dissipation theorem of the electrons, $\Sigma_{j}^{<}(E)=-f_{j}(E)\left(\Sigma_{j}^{r}(E)-\Sigma_{j}^{a}(E)\right)$, where $f_{j}(E)=$ $1 /\left[\exp \left(\left(E-\mu_{j}\right) /\left(k_{B} T_{j}\right)\right)+1\right]$ is the Fermi function at temperature $T_{j}$ and chemical potential $\mu_{j}$. The nonlinear self-energies (hence the subscript n) $\Sigma_{n, j}^{r,<}(E)$ of the electrons arising from the Hartree and Fock diagrams under SCBA [31,32] are (for dot $j=0,1)$ :

$$
\begin{aligned}
\Sigma_{\mathrm{n}, j}^{r}(E)= & i \hbar Q^{2}\left\{-\sum_{k=0,1} D^{r}\left(0, z_{j}, z_{k}\right) \int G_{k k}^{<}(\hbar \omega) \frac{d \omega}{2 \pi}+\right. \\
& \int \frac{d \omega}{2 \pi}\left[G_{j j}^{r}(E-\hbar \omega) D^{>}\left(\omega, z_{j}, z_{j}\right)+\right.
\end{aligned}
$$
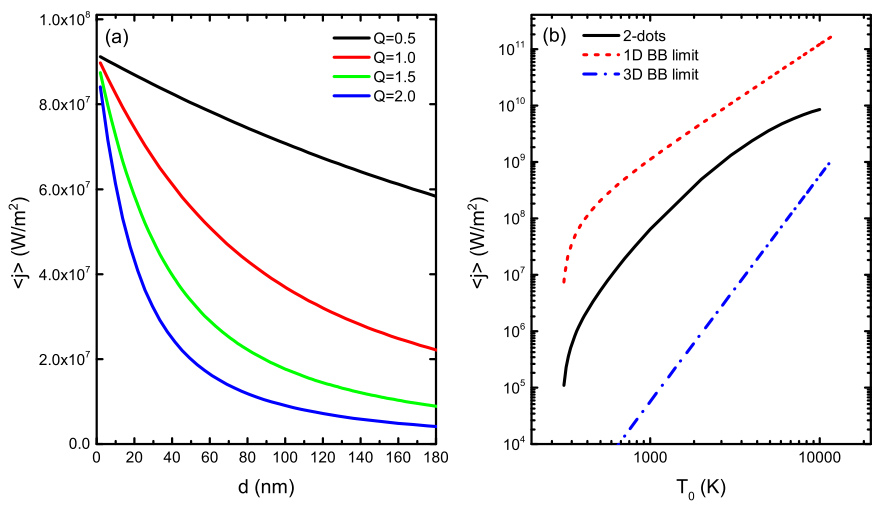

Fig. 2: (a) Heat current density $\langle j\rangle$ as a function of distance $d$ with different maximum charge $Q$ for the two-dot model. We set the temperatures of the baths at $T_{0}=1000 \mathrm{~K}, T_{1}=300 \mathrm{~K}$, chemical potentials $\mu_{0}=0 \mathrm{eV}, \mu_{1}=0.02 \mathrm{eV}$, and onsite $v_{0}=0$, $v_{1}=0.01 \mathrm{eV}$, area $A=389.4(\mathrm{~nm})^{2}$, and the electron bath parameters $\Gamma_{0}=1 \mathrm{eV}, \Gamma_{1}=0.5 \mathrm{eV}, E_{0}=2.0 \mathrm{eV}, E_{1}=1.0 \mathrm{eV}$. (b) The temperature dependence of heat current density. Here, we set $T_{1}=300 \mathrm{~K}$ and vary $T_{0}$, with $d=35.5 \mathrm{~nm}$ and $Q=1 e$. Other parameters are the same as for Fig. 2(a).

$$
\begin{gathered}
\left.\left.G_{j j}^{<}(E-\hbar \omega) D^{r}\left(\omega, z_{j}, z_{j}\right)\right]\right\} \\
\Sigma_{\mathrm{n}, j}^{<}(E)=i \hbar Q^{2} \int \frac{d \omega}{2 \pi} G_{j j}^{<}(E-\hbar \omega) D^{<}\left(\omega, z_{j}, z_{j}\right) .
\end{gathered}
$$

The current formula, Eq. (6), can be further simplified using the solution of the Dyson equation and then taking the limit $c \rightarrow \infty$, given

$$
\langle j\rangle A=\int_{0}^{\infty} \frac{d \omega}{\pi} \hbar \omega\left|D_{01}\right|^{2} i\left(\Pi_{11}^{>} \operatorname{Im} \Pi_{00}^{r}-\Pi_{00}^{>} \operatorname{Im} \Pi_{11}^{r}\right),
$$

where $D_{01}=\left[\Pi_{00}^{r} \Pi_{11}^{r} / C-\left(\Pi_{00}^{r}+\Pi_{11}^{r}\right)\right]^{-1} ; C=\epsilon_{0} A / d$ is the capacitance.

We now discuss the results of the two-dot model. Figure 2(a) shows the heat current density $\langle j\rangle$ as a function of the two-dot separation $d$. A key parameter is the area $A$; we choose it to be close to the experimental values in Ref. 11. The results are insensitive to the onsite potentials $v_{j}$ and chemical potentials $\mu_{j}$, provided $\Gamma_{j}$ is large. We note that the energy current takes an exact scaling form, $\langle j\rangle A=F(x), x=A /\left(Q^{2} d\right)$, with the area $A$, distance $d$, and charge $Q$. Further analysis shows that $F(x) \propto x^{2}$ for small $x$ and approaches a constant for large $x$. Under the strong coupling limit, $k_{B} T_{j} \ll \Gamma_{j}$, in addition to a $T_{j}^{4}$ temperature dependence, the current is proportional to $A /\left(d^{2} Q^{4}\right)$ for small $x$ and $1 /\left(A \Gamma^{2}\right)$ for large $x$ [here $\left.\Gamma \approx \max \left(\Gamma_{0}, \Gamma_{1}\right)\right]$. The crossover from one type of behavior to the other type of behavior is controlled by $\Gamma \approx Q^{2} /(2 C)$. Alternatively, a length scale can be obtained from $D_{01}$, giving $\tilde{d}=-\epsilon_{0} A\left[1 / \Pi_{00}^{r}(0)+1 / \Pi_{11}^{r}(0)\right]$. The current decreases as $1 / d^{2}$ for large $d$ and saturates at the scale given by $\tilde{d}$ (about $40 \mathrm{~nm}$ with our choice of parameters) for small $d$.

This phenomenon is different from near-field radiative heat transfer results at a length scale less than $\hbar c /\left(k_{B} T\right)$ 
dominated by evanescent modes when the transverse component of the wave vector $q_{\perp}>\omega / c$. There are no evanescent modes in our $1 \mathrm{D}$ model since $q_{\perp}=0$ permanently. Besides, Figure 2(a) also shows large values of heat transfer. At the saturation value, the heat current density is approximately $9 \times 10^{7} \mathrm{~W} / \mathrm{m}^{2}$, which is about a thousand times larger than the black-body $(\mathrm{BB})$ limit of $5.6 \times 10^{4}$ $\mathrm{W} / \mathrm{m}^{2}$. This enhancement is at least one order of magnitude larger than a typical $\mathrm{PvH}$ theory prediction for metals, which is in the range of the hundreds. Compared to a one-dimensional Landauer formula (1D BB) result with perfect transmission, i.e. $1.1 \times 10^{9} \mathrm{~W} / \mathrm{m}^{2}$, our numbers are about 1/12-th of that upper limit 33. Such enhancement is mainly due to transverse confinement (there is only one transmission mode) and the small area $A$. The temperature dependence of the current density is plotted in Fig. 2(b). Asymptotically for large $T_{0}$ fixing $T_{1}$, the Stefan-Boltzmann law gives the fourth power of $T_{0}$ and the $1 \mathrm{D}$ BB limit gives a quadratic function of $T_{0}$.

The results presented above are for strong couplings, where $\Gamma_{j}$ is comparable to electron energy scale of order eV. For weak couplings, SCBA convergence is difficult. However, we observe the physics remains qualitatively the same if we do not do self-consistency. In such a framework, the photon self-energies $\Pi_{j j}^{>}$can be approximated by the fluctuation-dissipation theorem, assuming each dot is in local thermal equilibrium. Within this framework of approximation, we can recover a Caroli/Landauer formula 24 .

In summary, we have presented a fundamental theory of near-field heat transfer due to Coulomb interactions of the electrons that applies to distances approaching atomic lattice constants. This is different from the usual $\mathrm{PvH}$ theory where only the transverse, radiative field is considered. At ultra-short distances, we should consider charge fluctuations and Coulomb interactions through a scalar field, which give an additional channel of heat transfer not contained in the Poynting vector [Note that if the vector potential $\mathbf{A}=0$, magnetic induction $\mathbf{B}$ is zero, so the Poynting vector is zero]. The contributions from the scalar potential $\phi$ are not small at short distances [20] as compared to the usual $\mathrm{PvH}$ results which include only the Poynting vector term involving the vector field. The approach proposed here opens the way for the treatment of other geometries such a surface and a tip. Such calculations could resolve the controversies regarding the recent experiment [1]. Our approach can be interfaced with first principle calculations, thus enabling more rigorous predictions of near-field properties.

The authors thank Lifa Zhang for stimulating discussions and Han Hoe Yap for pointing out an error in an earlier version of the paper. This work is supported by FRC grant R-144-000-343-112 and MOE grant R-144-000349-112.

\section{REFERENCES}

[1] Planck M., Theorie der Wärmestrahlung (J. A. Barth, Leipzig) 1906 translated into English by M. Masius in M. Planck, The Theory of Heat Radiation, (Dover, New York, 1991).

[2] Polder D. and van Hove M., Phys. Rev. B, 4 (1971) 3303.

[3] Hargreaves C. M., Phys. Lett., 30A (1969) 491.

[4] Domoto G. A., Boenm R. F. and Tien C. L., J. Heat Transf., 92 (1970) 412.

[5] Ottens R. S., Quetschke V., Wise S., Alemi A. A., Lundock R., Mueller G., Reitze D. H., Tanner D. B. and Whiting B. F., Phys. Rev. Lett., 107 (2011) 014301.

[6] Volokitin A. I. and Persson B. N. J., Rev. Mod. Phys., 79 (2007) 1291.

[7] Shen S., Narayanaswamy A. and Chen G., Nano Lett., 9 (2009) 2909.

[8] Song B., Fiorino A., Meyhofer E. and Reddy P., AIP Advances, 5 (2015) 053503.

[9] Kim K., Song B., Fernández-Hurtado V., Lee W., Jeong W., Cui L., Thompson D., Feist J., Reid M. T. H., García-Vidal F. J., Cuevas J. C., Meyhofer E. and RedDy P., Nature, 528 (2015) 387.

[10] Song B., Thompson D., Fiorino A., Ganjeh Y., Reddy P. and Meyhofer E., Nature Nanotechnology, 11 (2016) 509.

[11] Kloppstech K., Könne N., Biehs S.-A., Rodriguez A. W., Worbes L., Hellmann D. and Kittel A., , (2015) arXiv:1510.06311.

[12] Rytov S. M., Theory of Electric Fluctuations and Thermal Radiation (Air Force Cambridge Research Center, Bedford, MA) 1953.

[13] Bimonte G., Emig T., Kardar M. and Krüger M., , (2016) arXiv:1606.03740.

[14] Janowicz M., Reddig D. and Holthaus M., Phys. Rev. A, 68 (2003) 043823.

[15] Mahan G. D., Appled. Phys. Lett., 98 (2011) 132106.

[16] Xiong S., Yang K., Kosevich Y. A., Chalopin Y., D' Agosta R., Cortona P. and Volz S., Phys. Rev. Lett., 112 (2014) 114301.

[17] Chiloyan V., Garg J., Esfarjani K. and Chen G., Nature Comm., 6 (2015) 6755.

[18] Cohen-Tannoudji C., Dupont-Roc J. and GrynberG G., Photons 85 Atoms, introduction to quantum electrodynamics (Wliley-VCH) 2004.

[19] Wingreen N. S. and Meir Y., Phys. Rev. B, 49 (1994) 11040.

[20] Yu R., Manjavacas A. and de Abajo F. J. G., , (2016) arXiv:1608.05767.

[21] Keller O., Quantum Theory of Near-Field Electrodynamics (Springer, Berlin) 2011.

[22] Guidry M., Gauge Field Theories, an introduction with applications (John Wiley \& Sons, Inc) 1991.

[23] The anti-normal order is because the creation operators annihilate the photon vacuum state, $a_{q}^{\dagger}|0\rangle=0$.

[24] Peng J., Yap H. H., Zhang G. and Wang J.-S., , (2017) in preparation.

[25] Haug H. and Jauho A.-P., Quantum Kinetics in Transport and Optics of Semiconductors (Springer) 1996.

[26] Wang J.-S., Wang J. and Lü J. T., Eur. Phys. J. B, 62 
(2008) 381.

[27] Wang J.-S., Agarwalla B. K., Li H. and Thingna J., Front. Phys., 9 (2014) 673.

[28] Aeberhard U., J. Computational Electronics, 10 (2011) 394.

[29] Bruus H. and Flensberg K., Many-Body Quantum Theory in Condensed Matter Physics, an introduction (Oxford Univ. Press) 2004.

[30] Hedin L., Phys. Rev., 139 (1965) A796.

[31] Lü J.-T. and Wang J.-S., Phys. Rev. B, 76 (2007) 165418.

[32] Zhang L., Lü J.-T., Wang J.-S. and Li B., J. Phys.: Condens. Matter, 25 (2013) 445801.

[33] Ben-Abdallah P. and Joulain K., Phys. Rev. B, 82 (2010) $121419(\mathrm{R})$. 